\title{
Non-exponential networks in production
}

\author{
Alexander Popov ${ }^{1}$, and Rustem Valiev ${ }^{2, *}$ \\ ${ }^{1}$ Mechanical Engineering Research Institute of the Russian Academy of Sciences (IMASH RAN), \\ Moscow, Russia \\ ${ }^{2}$ The Russian Foreign Trade Academy, Moscow, Russia
}

\begin{abstract}
The article describes the characteristics of special types of queuing systems. Special types of queuing systems are listed. The main methods for calculating of non-exponential queuing systems are given.
\end{abstract}

The main disadvantage in describing really functioning objects by exponential queuing networks (QNs) is the assumption of the exponential distribution of the service times of requirements in the individual nodes. As to the Poisson nature of the incoming flow, this assumption is in many cases confirmed. Otherwise, the description of non-exponential QN completely coincides with the description of the exponential networks.

In this article, brief descriptions of the main methods for calculating non-exponential QNs are given. We assume that the flow entering the network is of Poisson nature, and the distribution of service times can be arbitrary/random [1].

Consideration of multiplicative QNs begins with the enumeration of special types of queuing systems (QSs) [2]:

a) Exponential system $M / M / n / \infty$.

b) Erlang system $M / G / n / 0$.

c) System $M / G / \infty$.

d) System $M / G / 1 / \infty$ with the discipline of uniform distribution of an element.

Let us consider the last system. To provide services to each element entering the system, you need to perform random work, the volume of which is distributed according to the law $G(x)$.

If there is only one requirement in the system, the system element performs this work at a unit rate, and, therefore, the service time of the requirement is the same as the amount of its work.

If there is $n$ number of requirements in the system, then the rate of work for each of them is $1 / n$. The stationary probabilities of the $p_{i}$ number of requirements in such a system have the following form [3]

$$
p_{i}=(1-\rho) \rho^{i},
$$

\footnotetext{
*Corresponding author: rmvaliev@gmail.com
} 
where $\rho=\lambda M=\lambda \int_{0}^{\infty} x d G(x)-$ is a system boot.

In other words, the stationary probabilities of the states in the system $M / G / 1 / \infty$ with the discipline of the equal apportionment of the element coincide with the stationary probabilities of the states in the conventional $M / M / 1 / \infty$ system with the same load [4].

e) The inversion order of the service with the interruption of maintenance and after service in the $M / G / 1 / \infty$ system consists in the fact that each new requirement entering the system interrupts the service of the requirements on the device and starts itself to be served.

The after service of requirements also occurs in the order opposite to the entry, and begins with the moment at which the service was interrupted. And in this system the stationary state probabilities are given in the formula (1).

It turns out that the described QSs (let us call them QSs a) - e) preserve the Poisson nature of their flow, that is, the flow of the requirements exiting them will also be of the Poisson nature (in this case, in the QS $M / G / n / 0$, we need to consider a common flow consisting of both served and lost requirements).

This property is fundamental for the multiplicative QNs. The preservation of the Poisson nature of the flow holds for some other disciplines of service, but they are so peculiar that they will hardly ever find any practical application [4].

Open multiplicative QNs are described in exactly the same way as the Jackson or Kelly networks, but each node can be of type a) - e) (here, as already mentioned, a flow exiting from the node of $M / G / n / 0$ type forms both serviced and unserviced requirements) [5].

The stationary probabilities of the states of the whole network break up into a product of stationary probabilities states of individual nodes, i.e. have a multiplicative form. In other words, the calculation of the multiplicative QNs reduces to the calculation of individual nodes, and the calculation of individual nodes can be performed using formulas for the exponential QSs.

A similar result holds for the closed systems. If we assume that only nodes of type b) e) are present in the network, then the multiplicative form will also be true for inhomogeneous networks. Nevertheless, multiplicative QNs are a rare phenomenon in the study of real systems.

Let us consider the use of the Markov models. This method is based on the fact that an arbitrary distribution function $G(x)$ can be approximated with any degree of accuracy by the hyper-Erlang distribution.

Using the hyper-Erlang approximation and the method of fictitious phases, it is possible to construct a Markov process with continuous time and a discrete set of states that describes the functioning of the considered network in sufficient detail. However, as in the case of exponential QNs, further investigation of the process encounters serious computational problems. Nevertheless, the method gives good results for the simplest QNs of the two- or three-phase QS type.

The most commonly used method is the approximate one for calculating the QNs, the essence of which consists in replacing the real flows entering the separate nodes of the network with the Poisson ones, and then in calculating individual nodes with the Poisson incoming flows. The stationary probabilities of the states of the whole network are also represented as a product of the stationary probabilities of the states of the individual nodes.

Sometimes, to improve the accuracy of calculations, the incoming flows are replaced not by the Poisson ones, but by the Erlang, hyper-Erlang, or recurrent flows. The accuracy of calculating the QNs using this method usually increases with the complexity of the network, i.e. with the increase in the number of flows entering each node.

Another approximate method for calculating QNs is the diffusion approximation method. Its use is based on the use of a special class of random processes. This method 
gives good results for QNs, loading of all nodes of which is close to unity.

Finally, the method of statistical tests (Monte-Carlo method, statistical modeling method, simulation method) must be mentioned. This method is based on a complete simulation on the computer of the process of system operation and collection, and analysis of the statistical data obtained.

The main disadvantages of the method of statistical tests are, firstly, the need to write complex modeling programs, and, secondly, high labour inputs of the modeling process itself.

One of the advantages of the approximate methods for calculating the QNs (including the method based on the use of Markov models) is that the possibility of their application in conditions of incomplete information.

For instance, very often there is either expectation value, or the first two moments of service time of requirements in the nodes that are known. This is sufficient in a number of cases to obtain relatively accurate values of such characteristics of the QNs as stationary medium lengths of the queues at network nodes [1].

\section{References}

1. Gnedenko B.V., Kovalenko I.N., Introduction to Queueing Theory (Moscow, Nauka, 1966)

2. Popov A.M., Sotnikov V.N., Sulimov A.Yu., Mathematical models in economics (Monograph. Moscow.: INEP, 2016)

3. Novikov A.I., Economic-mathematical methods and models (Textbook. Moscow, 2017)

4. Popov A.M., Sotnikov V.N., DIE EKONOMIKO-MATHEMATISCHEN METHODEN UND DIE MODELLE. DIE MONOGRAFIE (Monograph. Germany, 2015)

5. Popov A.M., Sermavbrin S.V., Shepilov A.M., NON-EXPONENTIAL NETWORKS OF PRODUCTS. [Proceedings of the All-Russian Scientific and Technical Conference of the School-Seminar "Transmission, processing and display of information" Terskol, 2013]. Krasnodar, Ekoinvest, 2014, pp.35-37. 